\title{
Should MMMT still be treated with adjuvant taxane-based combination chemotherapy?
}

\author{
Viola Heinzelmann-Schwarz ${ }^{1,2}$ (1) - André B. Kind ${ }^{2} \cdot$ Marcus Vetter $^{2} \cdot$ Kenneth Russell $^{3} \cdot$ Siti Omar $^{1}$. \\ Andreas Schoetzau ${ }^{2} \cdot$ Kerstin Hoeck $^{2} \cdot$ Daniel Fink $^{4} \cdot$ Michael L. Friedlander $^{5} \cdot$ Neville F. Hacker $^{1}$
}

Received: 5 August 2019 / Accepted: 19 November 2019 / Published online: 28 January 2020

(C) The Author(s) 2020

\begin{abstract}
Background Malignant mixed Mullerian tumors of endometrial (MMMT-E) and ovarian (MMMT-O) origin are associated with poor prognosis. Suggestively epithelial-driven tumors, their treatment has shifted from anthracycline or ifosfamide-based towards taxane-based chemotherapy. It remains unclear whether this change associates with better outcomes.

Patients and methods A conjoined Australian and Swiss patient cohort of MMMT-E $(N=103)$ and MMMT-O $(N=17)$ was compared to patients with adenocarcinoma of the endometrium (EC, $N=172)$ and ovary $(\mathrm{OC}, N=189)$. Clinicopathological characteristics, FIGO stage, first-line treatment, and patient outcomes were analyzed. The generated hypothesis was verified in an US-American cohort with high-grade serous ovarian cancer (HGSOC, $N=1290)$ and MMMT-O $(N=450)$ using immunohistochemistry and next-generation sequencing.

Results Early stage I/II MMMT-E showed a survival plateau after 2.5 years, with no recurrence or death observed afterwards. Relapse-free survival was significantly worse in MMMT-E treated with platinum/taxanes $(P=0.024)$ compared to non-taxane regimen. Hypothesizing that also MMMT-O might benefit from an adjuvant non-paclitaxel regimen, a second independent cohort of MMMT-O and HGSOC patients was examined. p53 mutations dominated in both cancers with comparable frequency. PI3KCA and KRAS mutations were less frequent: they were more frequent in MMMT-O than in HGSOC $(P=0.015$ and $P=0.018$, respectively). MMMT-O responded better to a combination of carboplatin with anthracyclines than with taxanes $(73.9 \%$ vs. $39.4 \%)$.

Conclusion Early stage I/II MMMT-E patients have excellent prognosis if no recurrence has appeared within the first 2.5 years. In MMMT-E, platinum/anthracycline or ifosfamide regimen associated with better outcomes than platinum/taxanes regimens. This might also apply to MMMT-O.
\end{abstract}

Keywords Carcinosarcoma $\cdot$ Epirubicin $\cdot$ Ifosfamide $\cdot$ Paclitaxel $\cdot$ Ovarian cancer $\cdot$ Endometrial cancer

Viola Heinzelmann-Schwarz and André B. Kind contributed equally.

Electronic supplementary material The online version of this article (https://doi.org/10.1007/s00432-019-03091-y) contains supplementary material, which is available to authorized users.

Viola Heinzelmann-Schwarz

viola.heinzelmann@usb.ch

1 Gynecological Cancer Centre, Royal Hospital for Women, School of Women's and Children's Health, University of New South Wales, Sydney, NSW 2031, Australia

2 Gynecological Cancer Centre, Hospital for Women, University of Basel, Spitalstrasse 21, 4021 Basel, Switzerland

\section{Introduction}

Carcinosarcoma or malignant mixed Mullerian tumors (MMMT) are biphasic tumors that contain malignant mesodermal and epithelial components, in contrast to sarcomas, which contain exclusively malignant mesodermal elements (Kernochan and Garcia 2009). Typically, the metastatic sites mainly

Caris Life Sciences, Basel, Switzerland

4 Department of Gynecology, University Hospital Zurich, Zurich, Switzerland

5 Medical Oncology, Prince of Wales Hospital, Prince of Wales Clinical School, University of New South Wales, Sydney, NSW 2031, Australia 
consist of carcinoma elements (Silverberg et al. 1990). Due to their rareness, there are no large epidemiological studies available and data are in general inconclusive. It has been proposed that the epithelial and sarcomatous elements develop from the same stem cell, with the carcinoma component being undifferentiated and of a specific histotype, whilst the sarcomatous component shows a mixed homologous appearance (McCluggage 2002; Gorai et al. 1997). Relapse-free and overall survival in MMMT-E is poor, and treatment failure at the time of recurrence appears to be at distant sites (Gadducci et al. 2002; Gonzalez Bosquet et al. 2010; Yamada et al. 2000; Manolitsas et al. 2001; Vorgias and Fotiou 2010). Surgical therapy is the initial treatment modality and necessitates hysterectomy, bilateral salpingo-oophorectomy and at least pelvic lymphadenectomy (NCCN 2001). If metastatic disease is present, there is a need for full cytoreduction, which can be achieved in 57\% of patients, resulting in a significant improvement of median overall survival (OS 53.3 vs. 8.6 months) (Tanner et al. 2011).

The benefit of chemotherapy for MMMT-E has been clearly shown, with active substances being cisplatin, carboplatin, ifosfamide, anthracyclines and paclitaxel (Sutton et al. 2000, 2005). It has been proposed that FIGO Stage I patients should be treated with 4 cycles and Stage II-IV patients with 6 cycles of carboplatin and epirubicin (Manolitsas et al. 2001; BertonRigaud et al. 2014; Wolfson et al. 2007). A recent Cochrane review including 579 patients from two randomized studies compared adjuvant radiotherapy with combination chemotherapy (ifosfamide with paclitaxel versus ifosfamide alone) in recurrent Stage III/IV MMMT-E, finding an improved OS in the combination chemotherapy treatment arms (Sutton et al. 2000; Homesley et al. 2009; Galaal et al. 2011). The promising survival data from various studies and the reduced toxicity found in the carboplatin and paclitaxel combination treatments which are predominantly used in endometrial and ovarian adenocarcinoma, led several institutions, including our own, to change the chemotherapy regimen in MMMT-E to this more tolerable combination. However, there is no established consensus for therapeutic management in this patient group (Berton-Rigaud et al. 2014; NCCN 2001). With only a few prospective randomized controlled trials reported in MMMT$\mathrm{E}$, the optimal chemotherapy modality is yet to be determined, particularly in view of new targeted therapies.

Therefore, this study retrospectively analyzed the outcomes of a large cohort of MMMT-E to evaluate the response to various kind of adjuvant chemotherapies.

\section{Materials and methods}

\section{Cohort description and outcome analysis}

We reviewed all patients with MMMT-E treated with an adjuvant platinum-based chemotherapy in the Gynecological
Cancer Centre of the Royal Hospital for Women in Sydney, Australia and the Gynecological Cancer Centers of the University Hospitals Zurich and Basel, Switzerland between 1988 and $2014(N=103)$. For each case, at least 1 control of EC $(N=172)$ and OC $(N=189)$ undergoing adjuvant platinum-based therapy was matched by age and FIGO Stage from the equivalent databases available. Data of cases and controls were obtained using paper and electronic patient records, as available, and the following parameters were collected and stored in an in-house study database: histological diagnosis and histotype of tumor, depth of myometrial invasion and involvement adjacent tissues, lymphvascular space invasion, age, BMI, menopausal status, menstrual status, HRT, type and duration of symptoms, date of diagnosis (matched), Stage of disease (FIGO I/II and III/IV, matched), grade, surgical procedure and its duration, blood loss and complications, lymphadenectomy including number of nodes removed, residual disease after primary surgery, chemotherapy regimen, number of cycles, response to treatment, time to progression, radiation treatment and date of death/relapse or last follow-up. Time to progression was defined as the time between the first day of treatment and either radiologic evidence of progressive disease or the first day of second-line treatment, whichever came first. Follow-up for all patients after treatment was at least every 3 months for the first 2 years, at least every 6 months for the subsequent 3 years and thereafter yearly for another 5 years or until death.

\section{Next-generation sequencing and immunohistochemistry}

Due to the results retrieved from this retrospective analysis in MMMT-E, it was decided to continue along the lines also for MMMT-O. For this purpose, a prospective US-American cohort of 450 MMMT-O and 1290 high-grade serous ovarian cancers (HGSOC) which underwent immunohistochemistry (IHC) and next-generation sequencing (NGS) within a commercially CLIA-certified molecular profiling laboratory as referrals from 2008 to 2016 was used (Caris Life Sciences, Phoenix, AZ, USA). The tissue diagnoses were submitted based on pathological assessment of physicians who requested the assays and were further verified by a pathologist at the Caris Laboratory. IHC was performed on formalin-fixed paraffin-embedded tumor samples using commercially available detection kits, automated staining techniques (Benchmark XT, Ventana, Tucson, AZ, USA), antibodies against ERCC1 (Clone 8F1, Abcam), TUBB3 (Clone PRB-435P, BioLegend) and TOP2A (Clone 3F6, Leica Biosystems). ERCC1 loss was defined as $\leq 2+$ staining in less than $50 \%$ of tumor cells or $3+$ staining in less than $10 \%$ of tumor cells. TUBB3 loss was defined as less than $2+$ staining present in less than $30 \%$ of tumor cells. A 
positive result for TOP2A was reported if at least $1+$ staining was observed in $10 \%$ or more of tumor cells. Test results from the company's commercial biomarker database were obtained anonymously using a data extraction tool.

\section{Statistical analyses}

Descriptive statistics for study groups were presented using mean \pm standard deviation (SD) or counts and percentages as appropriate. $P$ values were calculated using T-tests or Fisher's exact tests. Time to event was analyzed using Cox regression with corresponding hazard ratios (HR), 95\% confidence intervals (CI), $P$ values and the Kaplan-Meier method. A $P$ value $<0.05$ is considered as significant. Evaluations were done using the statistical software $\mathrm{R}$ version 3.1.1. Biomarker expression was compared across histologic subtypes via unpaired $t$ tests using GraphPad software (GraphPad Software Inc, La Jolla, CA, USA).

\section{Results}

We analyzed 103 histologically confirmed MMMT-E cases against 378 controls, consisting of $172 \mathrm{EC}, 189 \mathrm{OC}$ and 17 MMMT-O. The clinicopathological characteristics showed statistically significant differences for death from disease, age, BMI, FIGO Stage, grade, histotype, type of chemotherapy, adjuvant radiotherapy, residual disease, and lymph node dissection $(P \leq 0.001$, Table 1$)$. Approximately, onethird of patients with MMMT-E (31.1\%) and MMMT-O $(35.5 \%)$ died due to their disease by the end of this study, in contrast to one tenth (9.9\%) of EC and one-fifth (19\%) of OC patients. Almost two-thirds (64.9\%) in the MMMTE study group and three quarters $(76.4 \%)$ of the EC cases were early FIGO Stage I/II patients, in contrast MMMTO (17.6\%) and OC (32.4\%). MMMT-O and OC patients were mainly advanced FIGO Stage III/IV cases $(82.4 \%$ and $67.6 \%$, respectively). Significantly more undifferentiated and high-grade cancers were found in MMMT-E and MMMT-O
Table 1 Clinicopathological characteristics of the Swiss/ Australian cohort

\begin{tabular}{|c|c|c|c|c|c|c|}
\hline & $\begin{array}{l}\text { MMMT-E } N=103 \\
21.4 \%\end{array}$ & $\begin{array}{l}\mathrm{EC} \\
N=172 \\
35.8 \%\end{array}$ & $\begin{array}{l}\text { MMMT-O N=17 } \\
3.5 \%\end{array}$ & $\begin{array}{l}\text { OC } \\
N=189 \\
39.3 \%\end{array}$ & $\begin{array}{l}\text { Overall } \\
P \text { value }\end{array}$ & $\begin{array}{l}N \\
481\end{array}$ \\
\hline \multicolumn{7}{|l|}{ Age (years) } \\
\hline Mean \pm SD & $68.8 \pm 11.2$ & $66.6 \pm 11.8$ & $68.2 \pm 12.0$ & $63.3 \pm 12.9$ & 0.001 & 481 \\
\hline \multicolumn{7}{|l|}{ BMI } \\
\hline Mean \pm SD & $28.9 \pm 7.8$ & $33.5 \pm 10.5$ & $26.5 \pm 6.7$ & $26.6 \pm 6.0$ & $<0.001$ & 274 \\
\hline FIGO stage & 94 & 165 & 17 & 185 & $<0.001$ & 461 \\
\hline Stage I/II & $61(64.9 \%)$ & $126(76.4 \%)$ & $3(17.6 \%)$ & $60(32.4 \%)$ & & 250 \\
\hline Stage III/IV & $33(35.1 \%)$ & $39(23.6 \%)$ & $14(82.4)$ & $125(67.6 \%)$ & & 211 \\
\hline Grade & 45 & 164 & 15 & 161 & & 385 \\
\hline Grade 3 & $37(82.2 \%)$ & $55(33.5 \%)$ & $14(93.3 \%)$ & $107(66.5 \%)$ & $<0.001$ & 213 \\
\hline Other & $8(17.8 \%)$ & $109(66.5 \%)$ & $1(6.7 \%)$ & $54(33.5 \%)$ & & 172 \\
\hline Histotype & 35 & 172 & 9 & 188 & $<0.001$ & 404 \\
\hline Serous & $10(28.6 \%)$ & $29(16.9 \%)$ & $4(44.4 \%)$ & $126(67.0 \%)$ & & 169 \\
\hline Other & $25(71.4 \%)$ & $143(83.1 \%)$ & $5(55.6 \%)$ & $62(33.0 \%)$ & & 235 \\
\hline Chemotherapy & 53 & 19 & 14 & 136 & $<0.001$ & 222 \\
\hline $\mathrm{P} / \mathrm{A}$ & $47(88.7 \%)$ & $6(31.6 \%)$ & $6(42.9 \%)$ & $20(14.7 \%)$ & & 79 \\
\hline $\mathrm{P} / \mathrm{T}$ & $6(11.3 \%)$ & $13(68.4 \%)$ & $8(57.1 \%)$ & $116(85.3 \%)$ & & 143 \\
\hline Adjuvant RT & 95 & 98 & 17 & 15 & $<0.001$ & 222 \\
\hline Yes & $62(65.3 \%)$ & $45(45.9 \%)$ & $0(0 \%)$ & $4(26.7 \%)$ & & 111 \\
\hline $\mathrm{RD}$ & 71 & 62 & 16 & 9 & $<0.001$ & 158 \\
\hline None & $59(83.1 \%)$ & $53(85.5 \%)$ & $7(43.8 \%)$ & $6(66.7 \%)$ & & 122 \\
\hline LND & 95 & 74 & 15 & 8 & $<0.001$ & 192 \\
\hline Any & $66(69.5 \%)$ & $43(58.1 \%)$ & $2(13.3 \%)$ & $5(62.5 \%)$ & & 116 \\
\hline n.d. & $29(30.5 \%)$ & $31(41.9 \%)$ & $13(86.7 \%)$ & $3(37.5 \%)$ & & 76 \\
\hline DOD & $32(31.1 \%)$ & $17(9.9 \%)$ & $6(35.3 \%)$ & $36(19.0 \%)$ & $<0.001$ & 91 \\
\hline
\end{tabular}

$E C$ endometrial cancer, $M M M T-E$ malignant mixed Mullerian tumors of the endometrium, MMMT- $O$ malignant mixed Mullerian tumors of the ovary, $O C$ ovarian cancer, $B M I$ body mass index, $P / A$ platinum/ anthracycline, $P / T$ platinum/taxol, $R T$ radiotherapy, $R D$ residual disease, $L N D$ lymph node dissection done ("any") or not done ("n.d."), $D O D$ death of disease; statistical significance given by $P$ values 
patients (82.2 and $93.3 \%$, respectively) than in EC and OC patients $(33.5 \%$ and $66.5 \%$, respectively). Mainly, mixed and endometrioid histotypes were found in the MMMT-E study group ("other", 71.4\%) and in MMMT-O (55.6\%) and EC $(83.11 \%)$ patients; whereas OC patients were predominately of serous $(66.7 \%)$. A total of 222 patients received adjuvant chemotherapy, hereby platinum and either different anthracyclines or ifosfamide were mainly given in MMMT-E (88.7\%) and less in EC (31.6\%), MMMT-O (42.7\%) and OC (14.7\%). In contrast, a platinum/taxane regimen was used most commonly in OC (85.3\%), MMMT-O (57.1\%), EC $(68.7 \%)$ and only in $11.3 \%$ in MMMT-E. Adjuvant radiotherapy was applied in $65.3 \%$ to MMMT-E, $45.9 \%$ to EC, and $26.7 \%$ to OC.

We compared the long-term outcome, expressed as the cumulative risk of relapse, over a period of 20 years regardless of FIGO Stage. The cumulative relapse risk initially increased for all four cancers, strongest for MMMT-O patients and to comparable extents for OC, EC, and MMMTE (Fig. 1). Intriguingly, however, the cumulative risk for MMMT-E patients remained stable, namely reaching a plateau after 2.5 years until the end of the observation period (20 years); whereas, it further increased for the three other cancers to differing extent over this time period. We speculated as to whether the observed divergent survival results for MMMT-E and EC was dependent on the FIGO Stage. The relapse-free survival of MMMT-E and EC were, therefore, compared for early Stage (FIGO I/II) (Fig. 2a) and late Stage (FIGO III/IV) (Fig. 2b) patients in a Kaplan-Meier presentation. Indeed, the relapse-free survival rate of early Stage MMMT-E patients decreased to a greater extent within

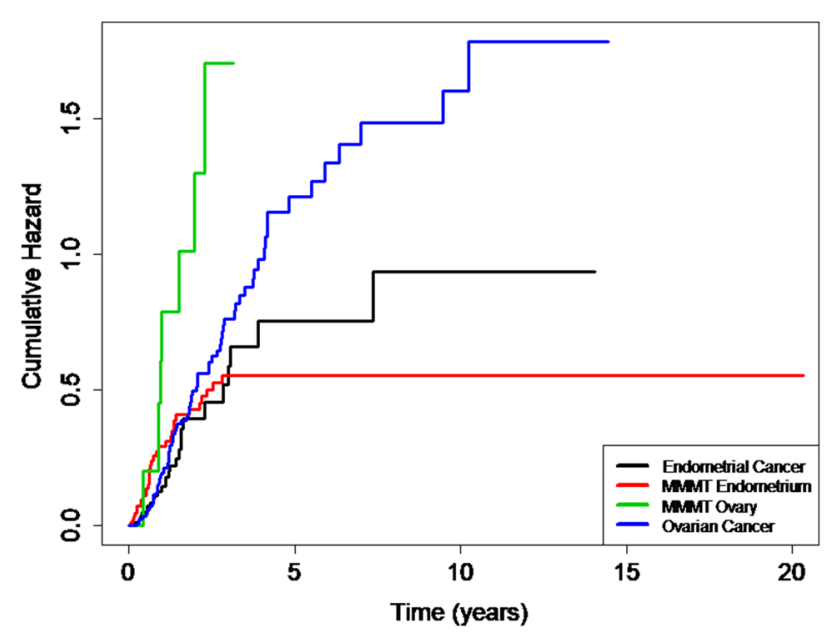

Fig. 1 Cumulative 20-year risk for relapse in MMMT-E, EC, MMMT-O and OC in our cohort. After an initial increase within 2.5 years, the cumulative hazard for relapse remained constant for MMMT-E (red line), whereas it continuously increased for endometrial cancer (black line), ovarian cancer (blue), and MMMT-O (green) over the years the first 2.5 years when compared to EC patients, but then remained stable at 0.75 , meaning that $75 \%$ of MMMT-E patients remained without any case of recurrence occurring for 20 years. The relapse-free survival rate of early FIGO stage EC patients was significantly different from that of the MMMT-E patients (strong intersection of the curves, test for proportional hazard $P<0.001$ ) and decreased to a lesser extent than in MMMT-E patients within the first 2.5 years
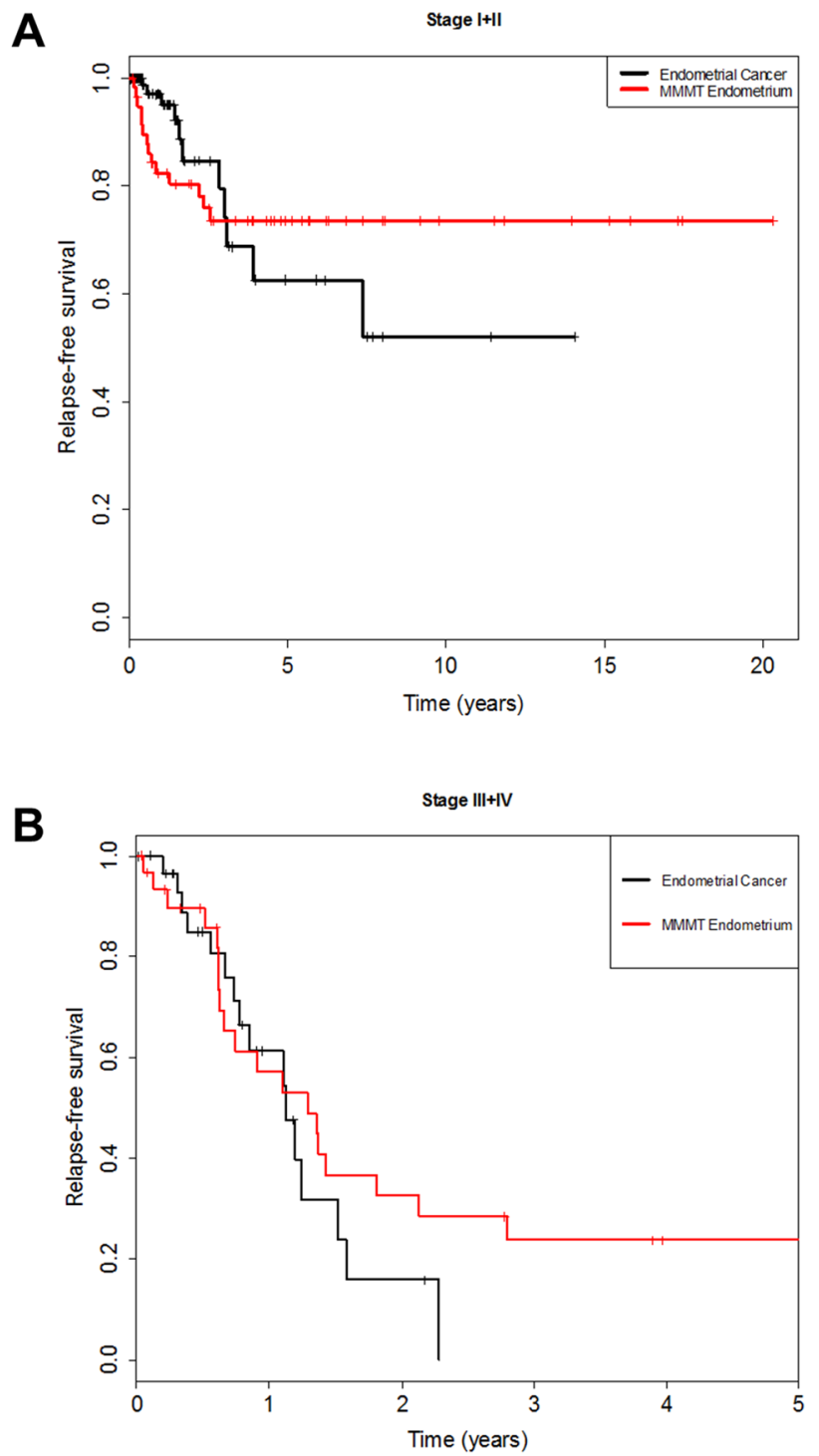

Fig. 2 Kaplan-Meier curve comparing early Stage (FIGO I/II) and late Stage (FIGO III/IV) MMMT-E and EC patients. Relapse-free survival for a early Stage MMMT-E patients (red line) reached plateau at 2.5 years and remains constant until the end of the observation period (20 years), whereas it further decreased for the respective EC patients with time (observation period 14 years); and b late Stage MMMT-E reached a plateau after 2.75 years, meaning that almost a quarter of these patients did not relapse after this time until the end of the observation period (5 years), whereas all EC patients relapsed already after 2.25 years. Hence, the early FIGO Stage I/II MMMT-E patients account for the observed plateau after 2.5 years 
but then further decreased within the subsequent 11.5 years. The respective relapse-free survival rates for advanced FIGO Stage MMMT-E and EC patients decreased to a comparable extent (proportional hazard $P=0.118$ ).

We also analyzed whether the choice of the chemotherapy regimen affected the observed favorable relapse-free survival of MMMT-E patients, regardless of FIGO Stage. Indeed, a significantly shorter time to relapse for patients receiving platinum and taxanes than those receiving platinum and anthracyclines or ifosfamide was observed (HR 4.69, CI $1.23-17.87, P=0.024$, Fig. 3). This indicates that MMMTE patients who received taxane-free platinum combination chemotherapy and particularly Stage FIGO I/II MMMT-E patients who do not relapse within the first 2.5 years have excellent long-term survival outcomes.

The increased risk of relapse in MMMT-E patients receiving taxane-based chemotherapy prompted us to also examine whether a similar effect could be present in MMMT-O. We, therefore, examined the mutational load and the protein expression of various drug targets in a large independent US-American cohort of MMMT-O $(N=450)$. MMMT-O and HGSOC $(N=1290)$ displayed a similarly high load of $p 53$ mutations ( $77.8 \%$ vs $80.2 \%$, Fig. $4 a$ ). In contrast, significant differences were found for $K R A S$ and $P I 3 K C A$ mutations, both being more frequent in MMMTO compared to HGSOC (KRAS: $5.7 \%$ vs $2.4 \%, P=0.015$; PI3KCA: $6.2 \%$ vs $3.3 \%, P=0.018)$.

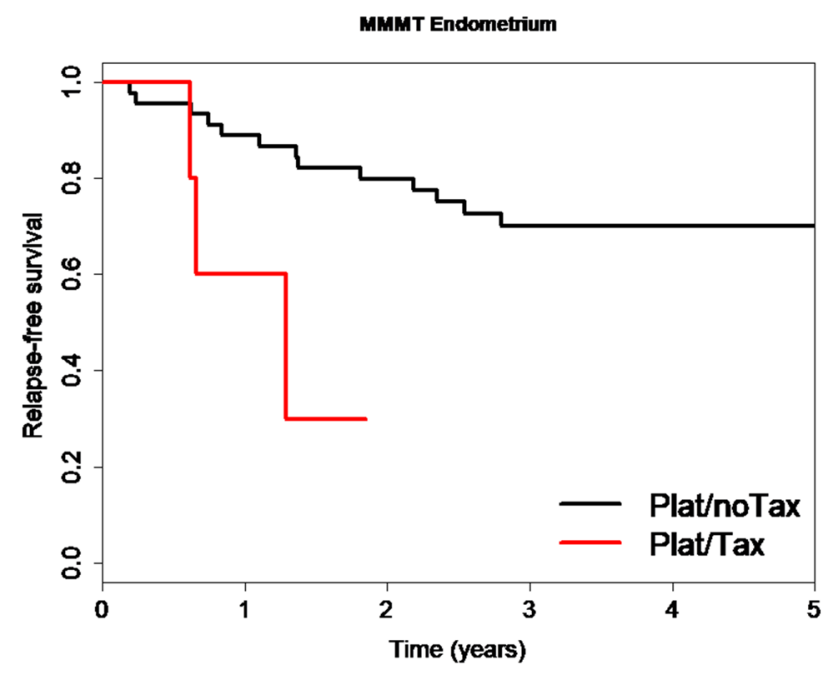

Fig. 3 Kaplan-Meier curve comparing relapse-free survival in MMMT-E patients subjected to platinum chemotherapy with or without taxanes. MMMT-E patients receiving platinum and taxanes (Plat/ Tax: red line) had a substantially worse relapse-free survival and shorter time to relapse (median: 1.29 years) than those with platinum and anthracyclines or ifosfamide (Plat/noTax: black line. Median time to relapse not available as median not reached) (HR 4.69, CI 1.23$17.87, P=0.024)$
The majority of proteins selected showed a statistically significant difference in the expression between MMMT-O and HGSOC (Supplement Table S1). These results, therefore, suggest that crizotinib (targeting ALK), cetuximab (EGFR), topotecan (TOPO1), anthracyclines (TOP2A), and alkylating agents such as ifosfamide (MGMT loss) would have a potential treatment benefit in MMMT-O. We next calculated a presumed sensitivity for the chemotherapy combination carboplatin/taxane compared to carboplatin/
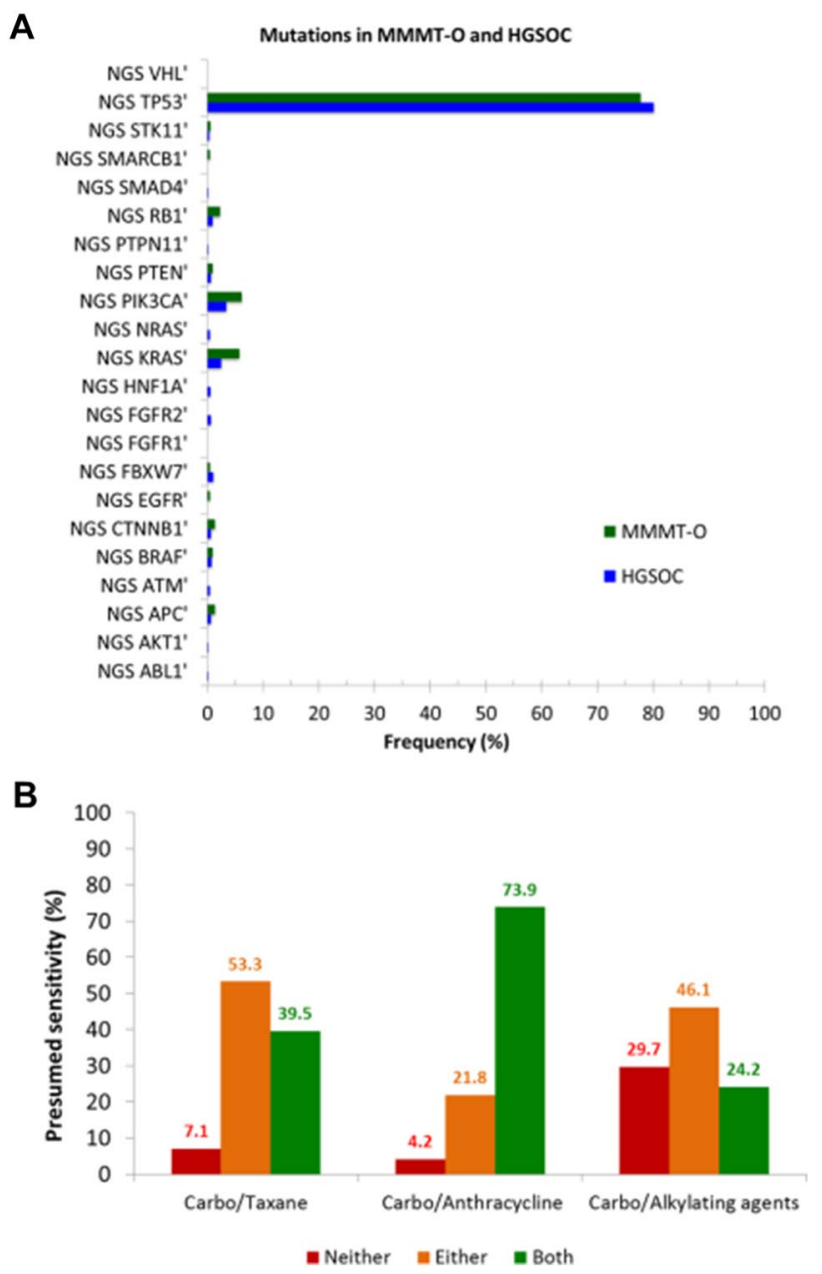

Fig. 4 Mutational load in MMMT-O and HGSOC and expression of chemotherapy target proteins in MMMT-O. a NGS-data showing the mutational load (frequency expressed in \%, $y$-axis) in MMMT-O (green) and HGSOC (blue). Mutation frequency expressed as percentage ( $y$-axis) is plotted against selected gene mutations ( $x$-axis). $K R A S$ and PIK3CA mutations were significantly more frequent in MMMT-O than in HGSOC ( $P=0.015$ and $P=0.018$, respectively). b Presumed chemosensitivity (expressed as percentage, $y$-axis) for MMMT-O based on the predictive biomarker expression for the combination of platinum with taxanes (left), with anthracyclines (middle), and with alkylating agents (right). Results of the prediction of sensitivity were based on whether none (red), one (orange) or both (green) of the biomarkers were expressed. Highest presumed sensitivity in MMMT-O was for the carboplatin/anthracycline combination (73.9\%; green, middle) 
anthracycline or carboplatin/alkylating agents in MMMTO. Hereby, the prediction of sensitivity to platinum/taxane combination was based on the loss of expression of ERCC1 and TUBB3 proteins, the prediction for the platinum/anthracycline combination was based on ERCC1 loss and TOP2A expression, and the prediction of platinum/alkylating agents combination was based on ERCC1 loss and MGMT loss. The results demonstrated for MMMT-O a presumed chemotherapy sensitivity of $39.5 \%$ for a combination of carboplatin/taxane, of $73.9 \%$ to a combination of carboplatin/ anthracyclines, and of $24.2 \%$ to a combination of carboplatin alkylating agents (Fig. 4b). These data suggest that not only MMMT-E but also MMMT-O patients may benefit from a taxane-free chemotherapy.

\section{Discussion}

It is increasingly important to define malignant diseases in relation to their genomic similarity instead of the organ of origin. This has been nicely shown in the M-PACT-trial from the US-National Cancer Institute, where the intention was to detect the molecular signature of diseases, rather than classify them by the tissue of origin. Subsequently, patient outcomes were examined according to mutations for $p 53$ and PIK3CA. Whilst the curves initially looked genetically driven, they diverged when the researchers looked at the tissue of origin (Schott et al. 2015).

MMMT of endometrial and ovarian origin share the same histological signature but their genetic similarity is still widely unknown. As MMMT-E and MMMT-O derive from different organs, they are not managed identical but due to the tissue of origin. One study suggests that MMMT-O and MMMT-E are different diseases in terms of their genetic landscape. In this study, 110 MMMT-O, 141 MMMT-E and 1587 OC of all histotypes where compared. TP53 was the most commonly mutated gene in all three cancers with 76.4\% in MMMT-O, 68.8\% in MMMT-E and 69\% in OC. Genetic alterations of PI3K/AKT/mTOR and MAPK pathways were noted to be similar in MMMT-O and OC but less frequent in MMMT-E $(p<0.001)$. In OC, the chance of having a BRCA1/2 mutation was highest compared to MMMT-E and MMMT-O (20\% and $18 \%$ vs. $9 \%$, respectively) (Mahdi et al. 2015). These data are similar to our own NGS analysis; however, we have 4-times more numbers of MMMT-O included and compared them to the most aggressive subtype of OC, HGSOC. Our own data in conclusion with the literature demonstrate a higher rate of KRAS and PI3KCA mutations in MMMT-O compared to HGSOC.

Since MMMT are known to be metaplastic carcinoma, they are no longer considered a subtype of sarcoma or managed as such. Instead, despite the lack of specific data, the management of MMMT has been extrapolated from studies of EC and OC (Berton-Rigaud et al. 2014; Cantrell et al. 2015). In the past 13 years, 9 GOG trials were performed in MMMT-E and MMMT-O. In total, 21 studies were found in our systematic literature search. Hereby, 16/21 were performed in MMMT-E only, 4/21 in MMMT-O only and $1 / 21$ in both types (Table 2). In total, 1214 patients were included in these heterogeneous studies. The largest GOG trial incorporated 206 patients, but most studies examined MMMT numbers below 100, which clearly limits its results. Cisplatin-based chemotherapy and adjuvant setup were most commonly studied (67\%), hereby the drug combination was mainly platinum plus paclitaxel or ifosfamide. Throughout these investigations, with mostly insufficient numbers of MMMT-E and MMMT-O patients, best response rate of a combination of carboplatin and paclitaxel was $62 \%$ and $55 \%$, respectively, and 5-year overall survival $62-88 \%$ and $30 \%$, respectively (Table 2 ). These data differ from our own results and might be due to the heterogeneity of the various cohorts and the small patient numbers in the published literature.

Targeted drug trials have been scarce for MMMT with only two studies examining the role of VEGF-directed therapy, one with pazopanib (MMMT-E), the other with aflibercept (MMMT-E and MMMT-O), both demonstrating only a minimal efficacy (Campos et al. 2014; Mackay et al. 2012). Our large retrospective case-control study with over 20 years of follow-up has clearly shown that the combination of carboplatin/taxanes is less efficient in MMMT-E. Our exploratory data further suggest that the same could be the case for MMMT-O.

The concept of personalized treatment is based upon NGS, CISH and IHC data on potentially targetable biomarkers that describe an individual molecular footprint of a tumor (Janssens et al. 2017). Using precision IHC, we found proteins that were differentially expressed between MMMT-E and MMMT-O. It has been hypothesized that these markers could be associated with the likelihood of response to chemotherapy. If confirmed, this may explain the poorer performance of the current standard treatments. Obviously, this is only a hypothesis generating observation and a carefully designed prospective clinical trial would be necessary to validate these findings.

The rarity of MMMT, in particular of MMMT-O, accounts for the fact that subgroup analyses in randomized controlled chemotherapy trials can rarely be performed because of a lack of statistical power. Secondly, different histopathological definitions of MMMT make it difficult to compare studies that focus on this group. Thirdly, many reports combine MMMT-E and MMMT-O with their organ-based adenocarcinoma counterparts. Treatment options tailored to the mutational driver $p 53$ or KRAS/ $P I 3 K C A$ as shown in our NGS analysis should, therefore, be considered in future studies to tailor treatment 


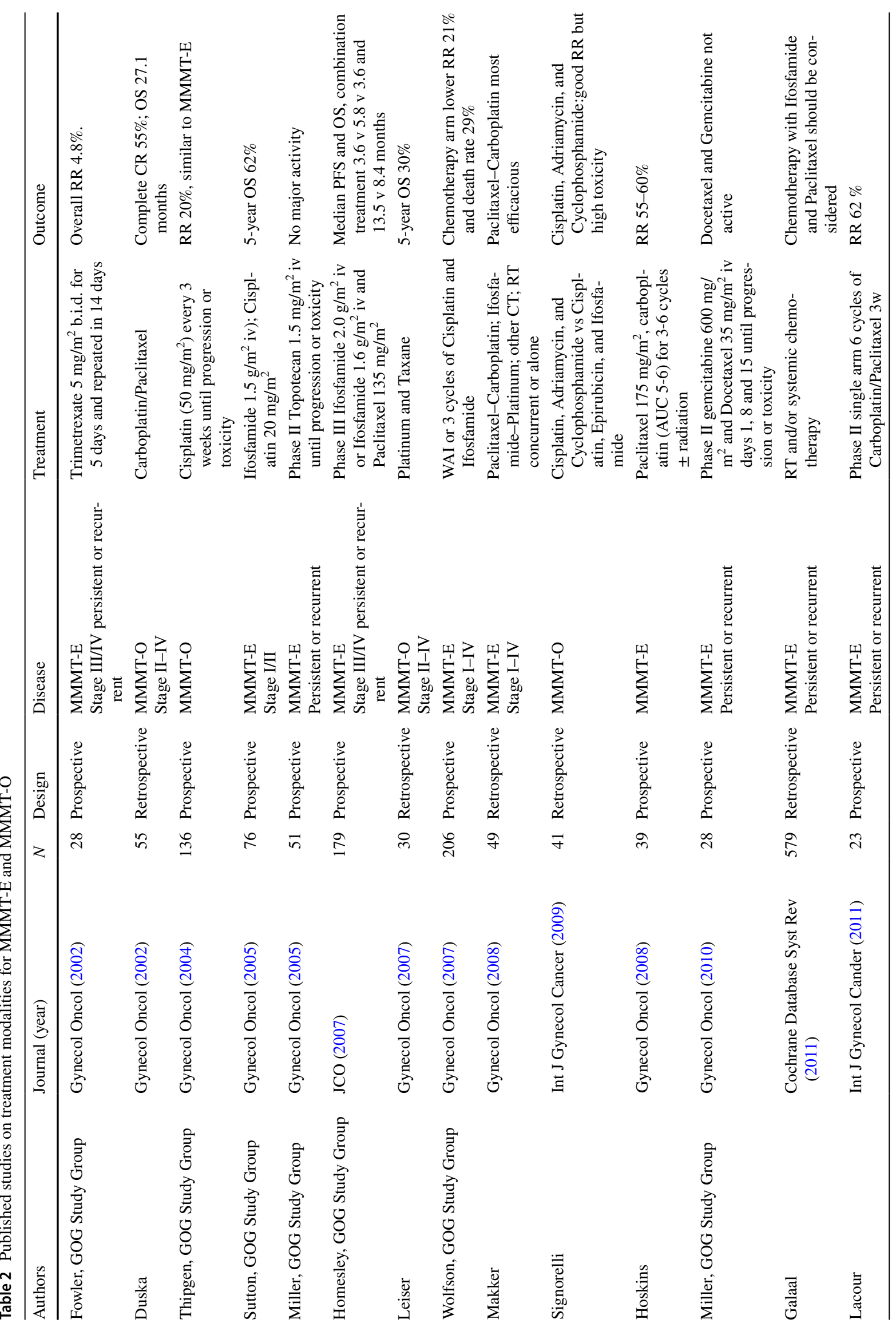




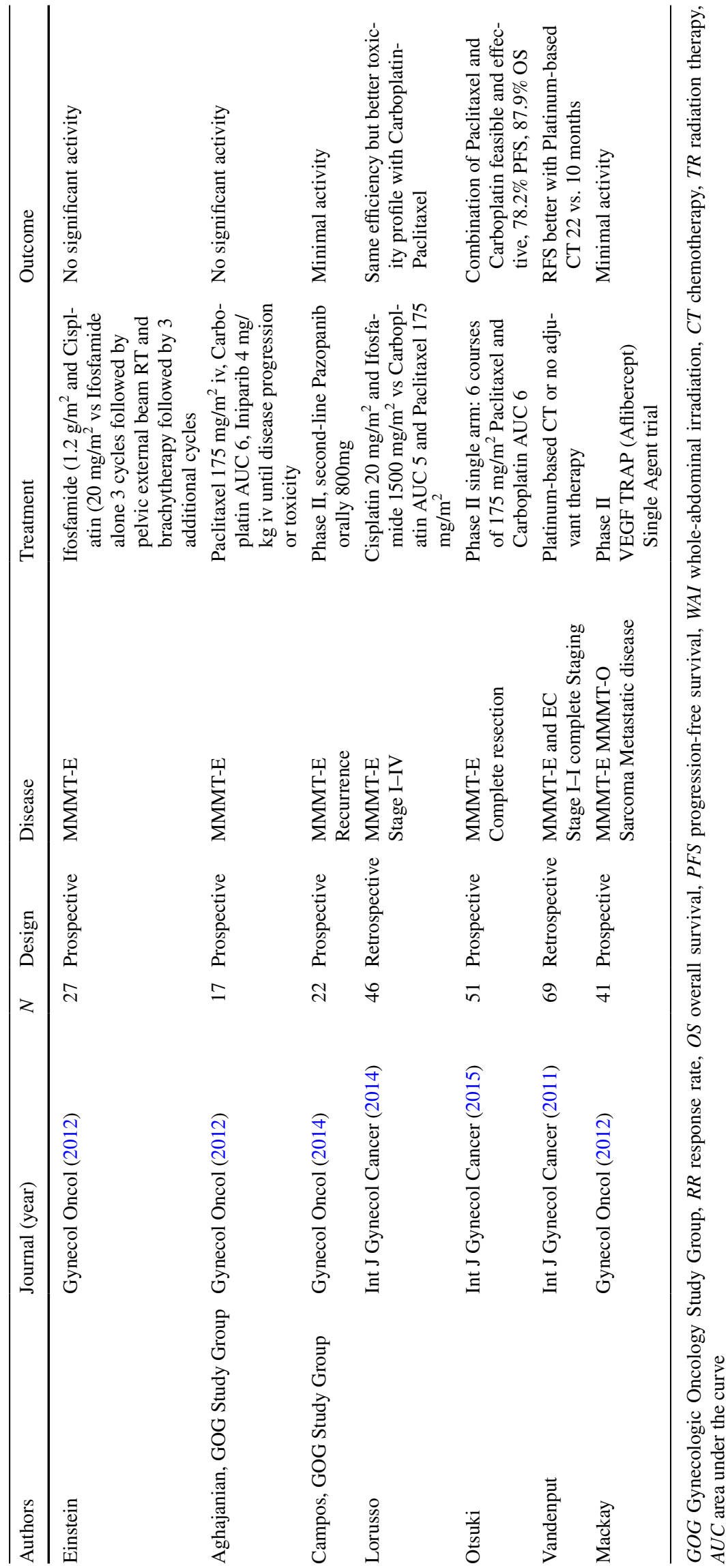


in relation to the genetic origin. For this, collaborative studies within trial networks performing whole genomic sequencing of these tumors is needed to identify potential targeted therapies for the future.

Acknowledgements We acknowledge the pathologists, medical and gynecological oncologists who were involved in the diagnosis and care of the examined patients at the Royal Hospital for Women, Sydney, the University Hospital Zurich and the University Hospital for Women, Basel.

Funding This work was supported by the Cancer Institute NSW (09/ CRF/2-02 to VHS.); RANZCOG (to VHS); William Maxwell Trust (to VHS); Swiss National Foundation (310030-156982 to VHS) and Oncosuisse (KFS_3013_08_2012 to VHS).

\section{Compliance with ethical standards}

Conflict of interest All authors declare that they have no conflict of interest.

Ethical standards The study was approved by the various institutional review boards (SPUK, Canton of Zurich, Switzerland, Hunter Area Research Ethics 04/04/07/3.04; South Eastern Sydney Illawarra HREC/ AURED Ref: 08/09/17/3.02, 08/09/17/3.02; StV06/2006).

Open Access This article is licensed under a Creative Commons Attribution 4.0 International License, which permits use, sharing, adaptation, distribution and reproduction in any medium or format, as long as you give appropriate credit to the original author(s) and the source, provide a link to the Creative Commons licence, and indicate if changes were made. The images or other third party material in this article are included in the article's Creative Commons licence, unless indicated otherwise in a credit line to the material. If material is not included in the article's Creative Commons licence and your intended use is not permitted by statutory regulation or exceeds the permitted use, you will need to obtain permission directly from the copyright holder. To view a copy of this licence, visit http://creativecommons.org/licenses/by/4.0/.

\section{References}

Aghajanian C, Sill MW, Secord AA et al (2012) Iniparib plus paclitaxel and carboplatin as initial treatment of advanced or recurrent uterine carcinosarcoma: a Gynecologic Oncology Group Study. Gynecol Oncol 126:424-427

Berton-Rigaud D, Devouassoux-Shisheboran M, Ledermann JA et al (2014) Gynecologic Cancer InterGroup (GCIG) consensus review for uterine and ovarian carcinosarcoma. Int J Gynecol Cancer 24(Suppl 3):S55-S60

Campos SM, Brady WE, Moxley KM et al (2014) A phase II evaluation of pazopanib in the treatment of recurrent or persistent carcinosarcoma of the uterus: a gynecologic oncology group study. Gynecol Oncol 133:537-541

Cantrell LA, Blank SV, Duska LR (2015) Uterine carcinosarcoma: a review of the literature. Gynecol Oncol 137:581-588

Duska LR, Garrett A, Eltabbakh GH et al (2002) Paclitaxel and platinum chemotherapy for malignant mixed müllerian tumors of the ovary. Gynecol Oncol 85:459-463

Einstein MH, Klobocista M, Hou JY et al (2012) Phase II trial of adjuvant pelvic radiation "sandwiched" between ifosfamide or ifosfamide plus cisplatin in women with uterine carcinosarcoma. Gynecol Oncol 124:26-30

Fowler JM, Blessing JA, Burger RA, Malfetano JH (2002) Phase II evaluation of oral trimetrexate in mixed mesodermal tumors of the uterus: a gynecologic oncology group study. Gynecol Oncol $85: 311-314$

Gadducci A, Sartori E, Landoni F et al (2002) The prognostic relevance of histological type in uterine sarcomas: a Cooperation Task Force (CTF) multivariate analysis of 249 cases. Eur J Gynaecol Oncol 23:295-299

Galaal K, van der Heijden E, Godfrey K et al (2011) Adjuvant radiotherapy and/or chemotherapy after surgery for uterine carcinosarcoma. Cochrane Database Syst Rev 2:CD006812

Gonzalez Bosquet J, Terstriep SA, Cliby WA et al (2010) The impact of multi-modal therapy on survival for uterine carcinosarcomas. Gynecol Oncol 116:419-423

Gorai I, Yanagibashi T, Taki A et al (1997) Uterine carcinosarcoma is derived from a single stem cell: an in vitro study. Int J Cancer $72: 821-827$

Homesley HD, Filiaci V, Markman M et al (2007) Phase III trial of ifosfamide with or without paclitaxel in advanced uterine carcinosarcoma: a Gynecologic Oncology Group Study. J Clin Oncol 25:526-531

Homesley HD, Filiaci V, Gibbons SK et al (2009) A randomized phase III trial in advanced endometrial carcinoma of surgery and volume directed radiation followed by cisplatin and doxorubicin with or without paclitaxel: a Gynecologic Oncology Group study. Gynecol Oncol 112:543-552

Hoskins PJ, Le N, Ellard S et al (2008) Carboplatin plus paclitaxel for advanced or recurrent uterine malignant mixed mullerian tumors. The British Columbia Cancer Agency experience. Gynecol Oncol 108:58-62

Janssens J, Gallagher WM, Dean A et al (2017) Tumor profilingdirected precision cancer therapy - comparison of commercial and academic clinical utility. Int J Surg Surg Porced 2:123

Kernochan LE, Garcia RL (2009) Carcinosarcomas (malignant mixed Müllerian tumor) of the uterus: advances in elucidation of biologic and clinical characteristics. J Natl Compr Canc Netw 7:550-556 (quiz 557)

Lacour RA, Euscher E, Atkinson EN et al (2011) A phase II trial of paclitaxel and carboplatin in women with advanced or recurrent uterine carcinosarcoma. Int J Gynecol Cancer 21:517-522

Leiser AL, Chi DS, Ishill NM, Tew WP (2007) Carcinosarcoma of the ovary treated with platinum and taxane: the memorial Sloan-Kettering Cancer Center experience. Gynecol Oncol 105:657-661

Lorusso D, Martinelli F, Mancini M et al (2014) Carboplatin-Paclitaxel versus Cisplatin-Ifosfamide in the treatment of uterine carcinosarcoma: a retrospective cohort study. Int J Gynecol Cancer 24:1256-1261

Mackay HJ, Buckanovich RJ, Hirte H et al (2012) A phase II study single agent of aflibercept (VEGF Trap) in patients with recurrent or metastatic gynecologic carcinosarcomas and uterine leiomyosarcoma. A trial of the Princess Margaret Hospital, Chicago and California Cancer Phase II Consortia. Gynecol Oncol 125:136-140

Mahdi H, Xiu J, Reddy SK (2015) Ovarian carcinosarcoma share similar molecular profile as ovarian serous carcinoma but not endometrial carcinosarcoma. J Clin Oncol 33:2015

Makker V, Abu-Rustum NR, Alektiar KM et al (2008) A retrospective assessment of outcomes of chemotherapy-based versus radiationonly adjuvant treatment for completely resected stage I-IV uterine carcinosarcoma. Gynecol Oncol 111:249-254

Manolitsas TP, Wain GV, Williams KE et al (2001) Multimodality therapy for patients with clinical Stage I and II malignant mixed Müllerian tumors of the uterus. Cancer 91:1437-1443 
McCluggage WG (2002) Uterine carcinosarcomas (malignant mixed Mullerian tumors) are metaplastic carcinomas. Int J Gynecol Cancer 12:687-690

Miller DS, Blessing JA, Schilder J et al (2005) Phase II evaluation of topotecan in carcinosarcoma of the uterus: a Gynecologic Oncology Group study. Gynecol Oncol 98:217-221

Miller BE, Blessing JA, Stehman FB et al (2010) A phase II evaluation of weekly gemcitabine and docetaxel for second-line treatment of recurrent carcinosarcoma of the uterus: a gynecologic oncology group study. Gynecol Oncol 118:139-144

NCCN (2001) Uterine neoplasms. Pract Guidel Oncol 2001:1-48

Otsuki A, Watanabe Y, Nomura $\mathrm{H}$ et al (2015) Paclitaxel and carboplatin in patients with completely or optimally resected carcinosarcoma of the uterus: a phase II trial by the Japanese Uterine Sarcoma Group and the Tohoku Gynecologic Cancer Unit. Int J Gynecol Cancer 25:92-97

Schott AF, Perou CM, Hayes DF (2015) Genome medicine in cancer: what's in a name? Cancer Res 75:1930-1935

Signorelli M, Chiappa V, Minig L et al (2009) Platinum, anthracycline, and alkylating agent-based chemotherapy for ovarian carcinosarcoma. Int J Gynecol Cancer 19:1142-1146

Silverberg SG, Major FJ, Blessing JA et al (1990) Carcinosarcoma (malignant mixed mesodermal tumor) of the uterus. A Gynecologic Oncology Group pathologic study of 203 cases. Int J Gynecol Pathol 9:1-19

Sutton G, Brunetto VL, Kilgore L et al (2000) A phase III trial of ifosfamide with or without cisplatin in carcinosarcoma of the uterus: a Gynecologic Oncology Group Study. Gynecol Oncol 79:147-153

Sutton G, Kauderer J, Carson LF et al (2005) Adjuvant ifosfamide and cisplatin in patients with completely resected stage I or II carcinosarcomas (mixed mesodermal tumors) of the uterus: a Gynecologic Oncology Group study. Gynecol Oncol 96:630-634

Tanner EJ, Leitao MM, Garg K et al (2011) The role of cytoreductive surgery for newly diagnosed advanced-stage uterine carcinosarcoma. Gynecol Oncol 123:548-552

Tate Thigpen J, Blessing JA, DeGeest K et al (2004) Cisplatin as initial chemotherapy in ovarian carcinosarcomas: a Gynecologic Oncology Group study. Gynecol Oncol 93:336-339

Vandenput I, Trovik J, Vergote I et al (2011) The role of adjuvant chemotherapy in surgical stages I-II serous and clear cell carcinomas and carcinosarcoma of the endometrium: a collaborative study. Int J Gynecol Cancer 21:332-336

Vorgias G, Fotiou S (2010) The role of lymphadenectomy in uterine carcinosarcomas (malignant mixed mullerian tumours): a critical literature review. Arch Gynecol Obstet 282:659-664

Wolfson AH, Brady MF, Rocereto T et al (2007) A gynecologic oncology group randomized phase III trial of whole abdominal irradiation (WAI) vs. cisplatin-ifosfamide and mesna (CIM) as postsurgical therapy in stage I-IV carcinosarcoma (CS) of the uterus. Gynecol Oncol 107:177-185

Yamada SD, Burger RA, Brewster WR et al (2000) Pathologic variables and adjuvant therapy as predictors of recurrence and survival for patients with surgically evaluated carcinosarcoma of the uterus. Cancer 88:2782-2786

Publisher's Note Springer Nature remains neutral with regard to jurisdictional claims in published maps and institutional affiliations. 